\title{
Acute generalized weakness due to thyrotoxic periodic paralysis
}

THE CASE: A 28-year-old First Nations man was transferred from a northern nursing station to the Health Sciences Centre in Winnipeg for investigation of a 1-day history of generalized weakness that began when he woke in the early morning. He had no fever, myalgia, or gastrointestinal, respiratory or cardiovascular symptoms, and no history of recent illness or known toxic ingestion. He did have a 3-year history of bilateral blindness, for which he had never sought medical attention. His family history was significant only for type 2 diabetes mellitus in his father and several maternal and paternal aunts and uncles.

On examination, he was alert, tachycardic (heart rate 140 beats $/ \mathrm{min}$ ) and afebrile. He had flaccid paralysis of his arms and legs (0/5) and could not lift his head off the bed. His wrist and digit strength were preserved (5/5). Deep tendon reflexes could not be elicited. Sensation to light touch and pinprick were normal. He had normal rectal tone. His visual acuity was decreased because of bilateral cataracts, but the cranial nerves were otherwise intact.

The patient had hypokalemia, with a serum potassium level of 1.6 (normal range 3.5-5.3) $\mathrm{mmol} / \mathrm{L}$, and his creatine phosphokinase level was slightly elevated at 328 (normal range 52-175) U/L. Results of other initial laboratory investigations were normal, including complete blood count, and renal and liver function tests.

An electrocardiogram revealed sinus tachycardia, a prolonged QT interval corrected to 664 ms, and ST-segment depressions inferiorly in leads II, III and avF (Fig. 1). A head CT revealed chronic bilateral retinal detachment as the cause of the patient's blindness but no intracranial abnormality to explain his weakness.

Twenty-four hours after symptom onset the patient still had hypokalemia (serum potassium level $1.9 \mathrm{mmol} / \mathrm{L}$ ), despite receiving a total of $200 \mathrm{mEq}$ of potassium orally and $160 \mathrm{mEq}$ parenterally. His distal lower limb strength had improved to $5 / 5$, with some return of strength in his shoulders and hips $(2 / 5)$. He was still unable to lift his head off the bed. Mild hyperreflexia was now present in his lower limbs.

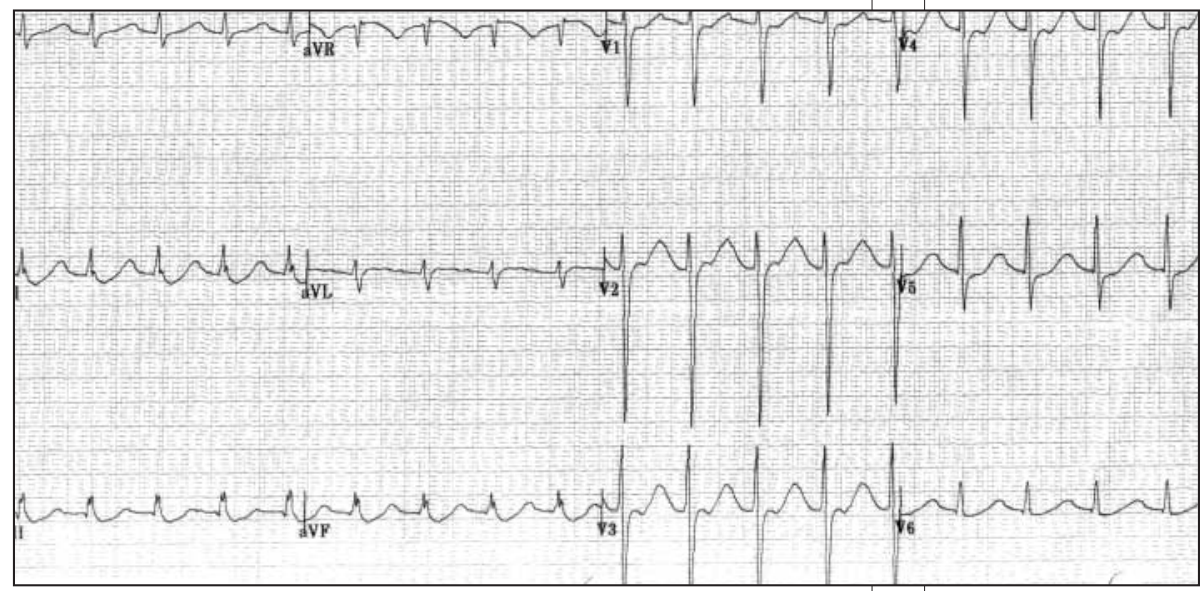

Dramatically, 2 hours later, the

patient was walking independently. His serum potassium level was normal $(5.0 \mathrm{mmol} / \mathrm{L})$, as was an electrocardiogram. Potassium replacement therapy was discontinued.

The results of thyroid function testing revealed thyrotoxicosis, with a thyroid stimulating hormone level of $<0.01$ (normal range 0.4-4.2) $\mathrm{mU} / \mathrm{L}$, an elevated free thyroxine level of 57 (normal range 9.7-25.7) pmol/L and a free triiodothyronine level of 18 (normal range 3.7-6.9) pmol/L. The patient agreed to treatment with propranolol followed by the addition of tapazole, but he declined radioactive iodine. At 10-month follow-up no paralysis had recurred.

7 hyrotoxic periodic paralysis (TPP) is a disorder characf terized by thyrotoxicosis, hypokalemia and predominantly proximal lower limb paralysis. Despite the female predominance of hyperthyroidism, TPP occurs most frequently in males in a ratio of $20: 1$, and $90 \%$ of patients are of Asian descent. ${ }^{1}$
Prodromal symptoms may consist of muscle stiffness or cramping. Sensation, as well as bulbar and respiratory muscle strength, remains intact. Reflexes may be decreased or absent.

Attacks may be precipitated by a high carbohydrate meal (secondary to insulin secretion) or physical exertion. In one series,
$84 \%$ of attacks occurred between 1 and 6 am. $^{2}$ Episodes are acute in onset and last 1-96 hours. As in this patient, symptoms and signs of hyperthyroidism may be subtle at initial presentation.

Electrocardiographic findings such as ST-segment depression with $\mathrm{T}$-wave flattening and the presence of $\mathrm{U}$ waves are 
typical of hypokalemia. Findings supportive of a diagnosis of TPP are sinus tachycardia, elevated QRS voltage and firstdegree AV block (sensitivity $97 \%$, specificity $65 \%$ ).

The exact pathophysiology of TPP is unknown. Thyroid hormone itself has a direct effect in stimulating the sodiumpotassium-adenosinetriphophatase (Na-K-ATPase) pump. Pump sensitivity to adrenergic stimulation may be higher in patients with TPP than in those with hyperthyroidism alone, resulting in intracellular potassium shift and subsequent

\section{Box 1: Differential diagnosis of acute weakness $^{1,6}$}

\section{Electrolyte disturbances}

- Hypo- or hyperkalemia

- Hypercalcemia

- Hypo- or hypernatremia

- Hypophosphatemia

- Hypo- or hypermagnesemia

Muscle disorders

- Channelopathies: periodic paralyses

- Metabolic defects of muscle (e.g., mitochondrial defects, impaired carbohydrate or fatty acid utilization)

- Myopathies: inflammatory, polymyositis, dermatomyositis, alcoholic myopathy, acute viral infection, parasitic polymyositis, Lyme disease, Legionnaire's disease

Neuromuscular junction disorders

- Myasthenia gravis

- Eaton-Lambert syndrome

- Organophosphate poisoning

- Botulism

Central nervous system disorders

- Transient ischemic attack (brainstem)

- Transient global cerebral ischemia

- Multiple sclerosis

Polyneuropathies

- Guillain-Barré syndrome

- Infections: poliomyelitis, West Nile virus

- Toxins

- Transverse myelitis hypokalemia. Some human leukocyte antigen subtypes (DRw8, A2BW22, AW19B17, $\mathrm{B} 5, \mathrm{BW} 46)$ and genetic mutations (KCNE3) have been associated with TPP. ${ }^{4}$

Potassium replacement therapy is the mainstay of treatment for TPP. It should be administered carefully because of the risk of rebound hyperkalemia and resulting cardiac complications. Recent literature has advocated instead for the use of $\beta$-blockers to reverse the adrenergic overstimulation of the $\mathrm{Na}-\mathrm{K}-\mathrm{ATP}-$ ase pump by elevated thyroid hormone levels. Evidence suggests that propranolol monotherapy reduces the duration of the TPP event and is associated with a lower risk of rebound hyperkalemia than potassium replacement therapy. ${ }^{5}$ Although $\beta$ blockers may be used in the acute setting, definitive management requires correction of the hyperthyroid state.

The differential diagnosis of acute weakness is summarized in Box 1. Broad categories to consider are electrolyte abnormalities, muscular disorders and central nervous system disorders at spinal cord, synapse and peripheral nerve levels. Specific causes of hypokalemic paralysis are summarized in Box 2 .

In summary, TPP should be suspected in patients presenting with acute proximal lower limb weakness. Management should include necessary resuscitative efforts, followed by $\beta$-blocker therapy and careful administration of potassium replacement therapy. Further attacks may be avoided by treatment of the hyperthyroid state.

\section{Cheryl Barnabé}

Resident

Department of Internal Medicine

University of Manitoba

Winnipeg, Man.
Box 2: Causes of hypokalemic paralysis ${ }^{1,5}$

Transcellular potassium shift

- Thyrotoxic periodic paralysis

- Familial hypokalemic periodic paralysis (autosomal dominant inheritance)

- Barium poisoning

- Insulin excess

Potassium deficit

Renal potassium loss

- Renal tubular acidosis Type 1: hereditary, toluene exposure, Sjögren's syndrome

Type 2: Fanconi's syndrome

- Hyperaldosteronism Primary (Conn's syndrome) Secondary: licorice ingestion

- Diuretics

Gastrointestinal potassium loss

- Celiac disease

- Infectious enteritis

- Short-bowel syndrome

\section{References}

1. Stedwell RE, Allen KM, Binder LS Hypokalemic paralyses: a review of the etiologies, pathophysiology, presentation, and therapy. Am $\mathcal{F}$ Emerg Med 1992;10:143-8.

2. Manoukian MA, Foote JA, Crapo LM Clinical and metabolic features of thyrotoxic periodic paralysis in 24 episodes. Arch Intern Med 1999;159:601-6.

3. Hsu Y, Lin Y, Chau T, Liou JT, Kuo SW, Lin SH. Electrocardiographic manifestations in patients with thyrotoxic periodic paralysis. $\mathrm{Am} F \mathrm{Med} \mathrm{Sci}$ 2003;326(3):128-32.

4. Salifu MO, Otah K, Carroll HJ, Ifudu O, Friedman EA, Oh MS. Thyrotoxic hypokalaemic paralysis in a black man. Q7M 2001;94:659-60.

5. Tassone $\mathrm{H}$, Moulin A, Henderson SO. The pitfalls of potassium replacement in thyrotoxic periodic paralysis: a case report and review of the literature. 7 Emerg Med 2004;26(2):157-61.

6. Kasper DL, Braunwald E, Fauci A, Hauser S, Longo D, Jameson JL, editors. Harrison's principles of internal medicine. 16th ed. New York: McGraw-Hill; 2005 